\title{
Acupotomy Alleviates Energy Crisis at Rat Myofascial Trigger Points
}

\author{
Yi Zhang $\mathbb{D},{ }^{1}$ Ning-Yu Du, ${ }^{2}$ Chen Chen, ${ }^{1}$ Tong Wang, ${ }^{1}$ Li-Juan Wang, ${ }^{1}$ Xiao-Lu Shi $\left(\mathbb{D},{ }^{3}\right.$ \\ Shu-Ming Li, ${ }^{4}$ and Chang-Qing Guo ${ }^{1}$ \\ ${ }^{1}$ School of Acupuncture-Moxibustion and Tuina, Beijing University of Chinese Medicine, Beijing 100029, China \\ ${ }^{2}$ Center for Early Childhood Development, Shijiazhuang Maternal and Child Health Care Hospital, Shijiazhuang 050051, China \\ ${ }^{3}$ Beijing Key Laboratory of TCM Basic Research on Prevention and Treatment of Major Disease, Experimental Research Center, \\ China Academy of Chinese Medical Sciences, Beijing 100700, China \\ ${ }^{4}$ Department of Pain Medicine, Beijing Hospital of Traditional Chinese Medicine, Capital Medical University, \\ Beijing 100010, China
}

Correspondence should be addressed to Yi Zhang; zhangyi.acupotomology@gmail.com

Received 20 November 2019; Accepted 18 January 2020; Published 28 February 2020

Academic Editor: Manel Santafe

Copyright (c) 2020 Yi Zhang et al. This is an open access article distributed under the Creative Commons Attribution License, which permits unrestricted use, distribution, and reproduction in any medium, provided the original work is properly cited.

\begin{abstract}
The aim of this study was to determine the effects of acupotomy on energy crises in rat trigger points (TrPs) by measuring mechanical pain thresholds (MPTs) and levels of acetylcholinesterase (AChE), free sarcoplasmic calcium $\left(\mathrm{Ca}^{2+}\right)$, adenosine $5^{\prime}$ triphosphate (ATP), adenosine $5^{\prime}$-monophosphate (AMP), substance P (SP), and calcitonin gene-related peptide (CGRP) in rat muscle $\operatorname{TrP}$ tissue. Male Sprague Dawley rats $(n=32)$ were randomly divided into four groups: control, TrP, acupotomy, and lidocaine injection. Enzyme-linked immunosorbent assays were used to measure AChE, and free sarcoplasmic $\mathrm{Ca}^{2+}$ concentrations were determined by fluorescent staining with Fura-2 AM; high-performance liquid chromatography was used to measure ATP and AMP, and SP and CGRP were evaluated by immunohistochemistry. Compared with the control group, free sarcoplasmic $\mathrm{Ca}^{2+}$, AMP, SP, and CGRP were higher in the model group, while MPT, AChE, and ATP were lower. Treatment with acupotomy or lidocaine injection reduced free sarcoplasmic $\mathrm{Ca}^{2+}, \mathrm{SP}$, and CGRP and increased MPTs and AChE levels compared with the model group. However, only acupotomy also led to decreased AMP and increased ATP levels relative to the model group. We conclude that acupotomy can alleviate energy crises at $\operatorname{TrPs}$.
\end{abstract}

\section{Introduction}

Myofascial pain syndrome (MPS) is a group of clinical disorders characterized by chronic pain arising from soft tissue, associated with one or multiple trigger points ( $\mathrm{TrPs}$ ) [1]. Myofascial TrPs are irritable points characterized by taut skeletal muscle bands. They can cause soft tissue pain that is activated by pressing, pulling, or overuse. TrPs can contribute to local pain, referred pain, and local twitch responses. They can also decrease muscle strength, work endurance, and coordination as well as cause other types of muscle dysfunction. TrPs are also associated with autonomic nerve manifestations, such as hyperhidrosis and arrector pili muscle response. Among patients with soft tissue pain, 20\%$95 \%$ suffer from MPS [2].
Although the exact nature of TrPs is unknown, Simons proposed the energy crisis hypothesis, which was subsequently developed to generate the integrated hypothesis $[3,4]$. It contends that contributing factors lead to the malfunction of motor endplates and excessive acetylcholine (ACh) leaking from the endplates, causing continuous depolarization of muscle cell membranes and calcium $\left(\mathrm{Ca}^{2+}\right)$ release from the sarcoplasmic reticulum, which cannot be reabsorbed. Therefore, the sarcoplasm continually has high $\mathrm{Ca}^{2+}$ concentrations, causing persistent muscle fiber contractions and the formation of taut intramuscular bands that can be palpated [5]. Continuous muscle contraction leads to local hypoxia and hypermetabolism, resulting in local energy crises and the release of sensitizing substances such as 5hydroxytryptamine, histamine, bradykinin, and substance $\mathrm{P}$ 
(SP). This stimulates nerve endings, causing pain and sympathetic neuronal symptoms [6].

Acupotomy, also referred to as miniscalpel-needle acupuncture, is a fairly recent development in the ancient practice of acupuncture. Acupotomy involves a combination of acupuncture needle insertion and surgical incision and is effective for relieving TrPs [7]. While TrPs are common sites for acupotomy, the mechanism underlying the effects of acupotomy on TrPs remains unclear. In this study, mechanical pain thresholds (MPTs) and levels of acetylcholinesterase (AChE), free sarcoplasmic $\mathrm{Ca}^{2+}$, adenosine $5^{\prime}$ triphosphate (ATP), adenosine $5^{\prime}$-monophosphate (AMP), calcitonin gene-related peptide (CGRP), and SP were evaluated in rat TrPs, and the effects of acupotomy on these indicators were compared with the effects of local lidocaine injection. The results provide a theoretical basis for different interventions to treat TrPs, including needle insertion at ashi points as described in TCM.

\section{Materials and Methods}

2.1. Animals. Male specific pathogen-free Sprague Dawley rats $(n=32$, 9 weeks old, weight $300 \pm 15 \mathrm{~g}$ ) were provided by Beijing Vital River Laboratory Animal Technology Co., Ltd. (animal batch number: SCXK(Jing)2016-0006). Rats were raised in the animal house at the Beijing University of Chinese Medicine with five rats per cage, free access to feed and water, natural lighting, indoor temperature of $22^{\circ} \mathrm{C} \pm 2^{\circ} \mathrm{C}$, humidity of $40 \%-60 \%$, and regular ultraviolet disinfection. The 32 rats were randomly divided into 4 groups ( $n=8$ each; control, TrP model, acupotomy, and lidocaine injection) using the random number table method. During the experiment, the rats were treated and handled with strict adherence to the standards set by the Ethical and Animal Research Committees, with approval from the National Natural Science Foundation (no. $81503653)$.

2.2. Experimental Reagents and Equipment. Experimental materials included acupotomes $(0.4 \times 40 \mathrm{~mm}$; ZYHY, Beijing, China), lidocaine hydrochloride injections (2\%; Yookon, Beijing, China), normal saline (Yookon), iodophor (Tiangen, Kunming, China), and disposable sterile syringes (Yuekang, Changzhou, China). Other reagents used were rat AChE enzyme-linked immunosorbent assay (ELISA) kit (CSB-E11304r; Huamei, Wuhan), phosphate-buffered saline (PBS) solution (Hyclone, Logan, UT, USA), Dulbecco's modified Eagle's medium (DMEM; Hyclone), Krebs-Henseleit solution (in mmol/L: $\mathrm{NaCl}, 118.50 ; \mathrm{NaHCO}_{3}, 25.00$; $\mathrm{KCl}, 4.70 ; \mathrm{MgSO}_{4}, 1.20 ; \mathrm{KH}_{2} \mathrm{PO}_{4}, 1.20$; glucose, $11.00 ; \mathrm{CaCl}_{2}$, 1.80 ; $\mathrm{NaOH}$ to $\mathrm{pH} 7.4$ ), Fura-2 AM powder (F1221; Invitrogen), Fura-2 Calcium Imaging Calibration Kit (F6774; Invitrogen), $5^{\prime}$-adenosine triphosphate disodium salt hydrate (Sigma), sodium adenosine $5^{\prime}$ - monophosphate (Sigma), rat SP immunohistochemical kits (ab14184, GR206575-1; Abcam), and rat CGRP immunohistochemical kits (ab81887, GR291095-3; Abcam).
2.3. Model Preparation. The rats, except those in the control group, were prepared as $\operatorname{Tr}$ P models by applying "blunt striking injury and eccentric exercise" as previously described [5]. Briefly, the rats were anesthetized with $20 \%$ urethane solution injected into the abdominal cavity $(0.5 \mathrm{~mL} / 100 \mathrm{~g})$. Rats were then fixed onto a plank under a striker, and the middle of the left vastus medialis was marked as the target. The striker was allowed to fall freely from a height of $20 \mathrm{~cm}$ to hit the marked position. On the second day, the rats were placed on $a-16^{\circ}$ treadmill set, so that they ran downhill continuously for $90 \mathrm{~min}$, with the speed gradually rising to $16 \mathrm{~m} / \mathrm{min}$, once per week, for 8 weeks. After the 8th week, rats in the control and model groups were examined by electromyography to confirm TrP model establishment.

2.4. Interventions. Rats in the four experimental groups described above were treated as follows: (1) Control, no intervention; (2) TrP model, no intervention after model preparation; (3) Acupotomy, acupotomy was applied 1 week after TrP model preparation; and (4) Injection group, lidocaine injections given 1 week after model preparation (20\% urethane solution injected into the abdominal cavity at a dosage of $0.5 \mathrm{~mL} / 100 \mathrm{~g}$, iodophor application to the $\operatorname{TrP}$ in the vastus medialis, and injection of $0.5 \mathrm{~mL}$ of $1 \%$ lidocaine into the taut band once a week for 3 weeks). Acupotomy intervention was conducted once per week for 3 weeks. The specific operation conducted at each point was as follows: (1) insertion of an acupotome into a $\operatorname{TrP}$ in the vastus medialis to pierce the taut band; (2) insertion of an acupotome perpendicular to the belly muscle, with the edge of the acupotome parallel to the taut band; and (3) the acupotome was then withdrawn and the point pressed for $30 \mathrm{~s}$.

\subsection{Index Assessments}

2.5.1. Electromyography. Electromyography assessments were performed in the control and model groups. Rats were anesthetized by intraperitoneal injection of $20 \%$ urethane $(0.5 \mathrm{~mL} / 100 \mathrm{~g})$ and then fixed on a board. The skin was cut to expose the left vastus medialis, and an electrode $(0.3 \mathrm{~mm})$ was inserted into the tail as a reference electrode. For the control group, an electrode was slowly inserted into the middle of the vastus medialis, and another electrode was inserted longitudinally $3 \mathrm{~mm}$ away from the last electrode into the vastus medialis. Finally, the wound was sutured. The same procedure was performed for the TrP group, but local twitching was considered confirmation of insertion into the $\mathrm{TrP}$. Electromyography recordings were performed when the muscle was at rest (Z2J-MB-NCC08; NCC, Shanghai, China).

2.5.2. $\operatorname{Tr} P$ MPTs. After the last intervention, 32 rats were anesthetized by $20 \%$ urethane. Anesthesia induction was confirmed by squeezing their tails using a power of $600 \mathrm{~g}$ [8] to see if it caused stable abdominal muscle contraction. The mechanical sensitivity of rat $\operatorname{TrPs}$ was assessed using the Von 
Frey mechanical pain stimulator (Ugo Basile, Genomio, Italy) to stimulate the $\operatorname{TrP}$ skin area until ipsilateral hind limb movement occurred [9]. The MPT was defined as the minimum stimulus intensity that caused the movement of the hind limbs. TrP sensitivity was assessed on days 1, 2, 3, 5, and 7 , next week after the last intervention. For each measurement, MPTs were determined three times, with an interval of $1 \mathrm{~min}$ between each test, and the mean of the results was calculated.

2.5.3. Sample Collection. Samples were collected after behavioral assessment. Rats were deeply anesthetized using $20 \%$ urethane and then fixed on an operating table with the left vastus medialis fully exposed. An experienced physician confirmed the position of the TrP in the vastus medialis by lightly pressing the taut band using the thumb or forefinger. To collect the samples, the vastus medialis was divided at the marked $\operatorname{TrP}$, the fascia was carefully removed, and muscle tissue samples $(1 \times 0.2 \mathrm{~cm})$ were excised. After sample collection, 32 rats were sacrificed by anesthesia overdose.

2.5.4. AChE Elisa. Tissue samples were placed in $900 \mu \mathrm{L}$ PBS (pH 7.4), rapidly minced with ophthalmic scissors, and homogenized using an ultrasonic homogenizer (FS-100T; sxsonic, Shanghai, China) and vibration table (KJ-201C; Kangjian, Jiangsu, China). Tissue homogenates were centrifuged with $6,000 \mathrm{~g}$ at $4^{\circ} \mathrm{C}$ for $15 \mathrm{~min}$, and the supernatants were separated. AChE levels in the samples were tested by an AChE ELISA kit according to the manufacturer's protocol. Optical density (OD) was read at $450 \mathrm{~nm}$ on a microplate reader (Multiskan MK3; Thermo Fisher Scientific, Waltham, MA, USA). Concentrations were calculated according to a standard curve, using the CurveExpert 1.3 software (https://www.curveexpert.net/).

\subsubsection{Fluorescent Staining to Detect Free Sarcoplasmic $\mathrm{Ca}^{2+}$.} The basement membrane of isolated muscle tissue was carefully removed to expose the muscle fibers. Then, a $1 \mathrm{~cm}$ length of muscle tissue was cut along the direction of the muscle fibers and rinsed with PBS buffer to load with fluorescent dye. Fura-2 AM stock solution was prepared by dissolving Fura-2 AM powder (F1221, Invitrogen) in 20\% Pluronic F127 DMSO solution. The muscle tissue was incubated with fluorescent staining solution (final Fura-2 concentration was $2.5 \mu \mathrm{mol} / \mathrm{L}$ in DMEM solution) in the dark at the $37^{\circ} \mathrm{C}$ temperature. After 30 min loading, replace the fluorescent loading solution by $\mathrm{KH}$ solution and incubated another $5 \mathrm{~min}$ for twice at the $37^{\circ} \mathrm{C}$ temperature to remove unloaded Fura-2 AM. The fluorescence-stained samples in $\mathrm{KH}$ solution were then placed in $35 \mathrm{~mm}$ glass plates, and only the resting free sarcoplasmic $\mathrm{Ca}^{2+}$ in intact skeletal muscle fibers was measured using an Andor iXon3 EMCCD (Andor, Belfast, UK) with a 10x fluorescence objective lens. A Sutter DG4 Xenon lamp system (Sutter, Sacramento, CA, USA) was used as the excitation light source at $340 \pm 10 \mathrm{~nm}$ and $380 \pm 10 \mathrm{~nm}$, separately, with emitted light measured at $510 \pm 25 \mathrm{~nm}$. $\mathrm{Ca}^{2+}$ images were collected from three different positions for each sample, and three muscle fibers were analyzed in each image. The mean value for the muscle fibers was considered the $\mathrm{Ca}^{2+}$ level in the tissue sample. Background fluorescence without staining was also measured for each sample. Fluorescence intensities at 340 and $380 \mathrm{~nm}$ were corrected by background intensity. The fluorescence intensity ratio was determined using the 340 and $380 \mathrm{~nm}$ excitation wavelengths, and tissue $\mathrm{Ca}^{2+}$ concentration was subsequently calculated using the following formula [10]: $\left[\mathrm{Ca}^{2+}\right]=K_{\mathrm{d}} \times\left(R-R_{\min }\right) /\left(R_{\max }-R\right) \times S_{\mathrm{f} 2} /$ $S_{\mathrm{b} 2}$ (in the formula, $K_{\mathrm{d}}$ is the effective dissociation constant, $R$ is the fluorescence ratio, $S_{\mathrm{f} 2}$ is the proportionality coefficient for free dye measured at wavelength $\lambda_{2}$, and $\mathrm{Sb}_{2}$ is the proportionality coefficient for $\mathrm{Ca}^{2+}$-bound dye at $\lambda_{2}$ ). The values of $R_{\max }$ and $R_{\min }$ were the ratio values measured by Fura-2 Calcium Imaging Calibration Kit (F6774, Invitrogen).

2.5.6. ATP and AMP Measurement. Tissue samples were weighed and added to precooled $0.4 \mathrm{~mol} / \mathrm{L}$ perchloric acid $(5 \mu \mathrm{L} / \mathrm{mg})$ and then homogenized in an ultrasonic cell grinder (SCIENTZ-1200E; Scientz, Zhejiang, China). Homogenates were centrifuged at $10000 \mathrm{~g}\left(4^{\circ} \mathrm{C}, 10 \mathrm{~min}\right)$. Then, $100 \mu \mathrm{L}$ supernatant and $50 \mu \mathrm{L}$ perchlorate were added into a $1 \mathrm{~mol} / \mathrm{L}$ potash-methanol mixture (volume $4: 1$ ), and the mixture was centrifuged at $10,000 \mathrm{~g}\left(4^{\circ} \mathrm{C}, 10 \mathrm{~min}\right)$. Next, a $100 \mu \mathrm{L}$ aliquot of the supernatant was removed, diluted with $100 \mu \mathrm{L}$ water, and assessed by high-performance liquid chromatography. The conditions were as follows: mobile phase, $30 \mathrm{mM} \mathrm{NaH} \mathrm{PO}_{4}$ (pH 6.25), 3\% methyl alcohol; detection wavelength, $254 \mathrm{~nm}$; sample size, $20 \mu \mathrm{L}$; column temperature, room temperature; sample temperature, $4^{\circ} \mathrm{C}$.

2.5.7. Immunohistochemical Analysis of SP and CGRP. Muscle biopsies were fixed in $10 \%$ formalin and embedded in paraffin. After sectioned at $4 \mu \mathrm{m}$ thickness, sections were dehydrated in xylene, rehydrated in graded alcohol, and then rinsed with PBS. Then, the antigen was retrieved by $\mathrm{mi}-$ crowave $(800 \mathrm{~W})$ in $0.01 \mathrm{M}$ citric acid buffer ( $\mathrm{pH}$ 6.0) for $10 \mathrm{~min}$, washed with PBS three times, and then placed in the $3 \% \mathrm{H}_{2} \mathrm{O}_{2}$ at room temperature for $60 \mathrm{~min}$. The antibodies were diluted with PBS buffer. Slides were incubated $\left(4^{\circ} \mathrm{C}\right)$ overnight with rabbit anti-SP $(1: 500$; Abcam $)$ or rabbit antiCGRP (1:200; Abcam) and then with the appropriate secondary biotinylated antibody (Boster) for $60 \mathrm{~min}$ at room temperature. The slides were then incubated with the avidinbiotinylated enzyme complex and then put into a peroxidase reaction solution containing diaminoaniline. Afterward, fresh DAB was added. Then, the slides were washed with PBS, counterstained in hematoxylin, blued in running water, dehydrated with gradient alcohol, cleared with xylene, and mounted with neutral gum. Areas positive for SP or CGRP in randomly selected fields of vision (original magnification $\times 200$ ) were counted using Image-Pro Plus 6.0 (Media Cybernetics, Rockville, MD, USA). The resulting data were collected as immunohistochemical staining-positive signal cumulative optical density (IOD) values, which represent the 
average positive area. These IOD values were used as quantitative measures of tissue expression levels.

2.6. Statistical Analysis. Data were analyzed using the SPSS 22.0 statistical software (v22.0; IBM Corp., Armonk, NY, USA). For normally distributed data with homogeneous variance, analysis of variance (ANOVA) was used to compare four groups. For non-normally distributed data, the Kruskal-Wallis test was used. All data are expressed as mean \pm standard deviation. $P<0.05$ and $P<0.01$ were considered to indicate significant and very significant differences, respectively (Figure 1).

\section{Results}

3.1. Electromyography of Model Group. Electromyography was recorded at $10 \mathrm{~ms} / \mathrm{D}$ and $20 \mu \mathrm{V} / \mathrm{D}$. Figure $2(\mathrm{a})$ shows a representative typical endplate potential, which turns negative from baseline. Figure 2(b) shows the fibrillation potential, which turns positive from baseline and then turns negative. This suggests the presence of TrPs in the vastus medialis of the model group rats [6].

3.2. Acupotomy Effect on $\operatorname{Tr} P$ MPTs. Compared with the control group, the MPT of the model group decreased on the 1 st, 2 nd, 3rd, 5th, and 7 th days after intervention $(P<0.05$, Figure 3). Moreover, compared with the model group, the MPT increased in the acupotomy group on the 1st, $2 \mathrm{nd}, 3 \mathrm{rd}$, 5 th, and 7 th days after intervention $(P<0.05$, Figure 3$)$. Further, relative to the model group, the MPT was higher in the lidocaine injection group on the $1 \mathrm{st}, 2 \mathrm{nd}, 3 \mathrm{rd}, 5 \mathrm{th}$, and 7 th days after intervention $(P<0.05$, Figure 3$)$. Comparisons between the acupotomy and injection groups showed no significant difference in MPTs at any time point tested $(P>0.05$, Figure 3).

3.3. Acupotomy Effect on AChE. AChE levels were significantly lower $(P<0.05)$ in the model group than the control group $(P<0.05)$. However, AChE levels in the acupotomy $(P<0.05)$ and injection $(P<0.05)$ groups were significantly increased compared with the model group (Figure 4 , Table 1). There was no significant difference between the acupotomy and injection groups $(P>0.05$, Figure 4 , Table 1). These data show that acupotomy can increase AChE levels in TrPs.

3.4. Acupotomy Effect on Free Sarcoplasmic $\mathrm{Ca}^{2+}$. Free sarcoplasmic $\mathrm{Ca}^{2+}$ in the model group was significantly higher than in the control group $(P<0.05)$. Relative to the model group, free sarcoplasmic $\mathrm{Ca}^{2+}$ in the acupotomy and injection groups was decreased significantly (both $P<0.05$, Figure 5, Table 2). There was no significant difference between the acupotomy and injection groups $(P>0.05$, Figure 5, Table 2). These findings demonstrate that acupotomy can reduce free sarcoplasmic $\mathrm{Ca}^{2+}$ levels in TrPs.
3.5. Acupotomy Effect on ATP and AMP. ATP levels in the model group were significantly lower than those in the control group $(P<0.05)$. Relative to the model group, ATP increased significantly in the acupotomy group $(P<0.05$, Figure 6, Table 3); however, there was no significant difference between the model and injection groups $(P>0.05$, Figure 6, Table 3 ). These findings indicate that acupotomy can enhance ATP levels in TrPs.

AMP levels in the model group were significantly higher than those in the control group $(P<0.05)$, but those in the acupotomy group were significantly lower than those in the model group $(P<0.05$, Figure 6 , Table 3$)$. Again, there was no significant difference between the model and injection groups $(P>0.05$, Figure 6 , Table 3$)$. These results show that acupotomy can decrease AMP levels in TrPs.

3.6. Acupotomy Effect on SP and CGRP. In Figure 7, SP expression was yellowish brown in muscle fibers. SP levels were significantly higher in the model group than the control group $(P<0.05)$, but they were significantly lower in the acupotomy group than the model group $(P<0.05$, Figure 6 , Table 4). Furthermore, compared with the model group, SP levels were significantly lower in the injection group $(P<0.05$, Table 4$)$. There was no significant difference between the acupotomy and injection groups $(P>0.05$, Figure 7, Table 4). Hence, our findings indicate that acupotomy can reduce $\mathrm{SP}$ levels in $\mathrm{TrP}$ muscle tissue.

In Figure 8, CGRP expression was yellowish brown in muscle fibers. CGRP levels were significantly higher in the model group than the control group $(P<0.05)$, but those in the acupotomy $(P<0.05)$ and injection $(P<0.05)$ groups were significantly lower than those in the model group. There was no significant difference in CGRP content between the acupotomy and injection groups $(P>0.05$, Figure 8 , Table 4 ). These results suggest that acupotomy can reduce CGRP content in $\operatorname{TrP}$ muscle tissue.

\section{Discussion}

The results of this study demonstrate that the MPT, AChE, free sarcoplasmic $\mathrm{Ca}^{2+}$, ATP, AMP, SP, and CGRP levels were altered in rats after TrP model generation, and acupotomy improved these effects. Other than the changes in ATP and AMP levels, which were improved by acupotomy but not lidocaine injection, there were no significant differences between the effects of acupotomy and those of lidocaine injection.

4.1. Energy Crisis at Trigger Points. Despite several theories, the exact nature of TrPs remains unknown $[11,12]$; however, electrophysiological and histological evidence support the integrated hypothesis of the cause of TrPs [13], which contends that an abnormal increase in ACh causes increased energy consumption in TrPs. Specifically, motor endplates in active $\mathrm{TrPs}$ release excessive $\mathrm{ACh}$ in the resting state, resulting in continuous depolarization of muscle fibers. This causes the sarcoplasmic reticulum to release excessive $\mathrm{Ca}^{2+}$ into myoplasm, followed by continuous muscle fiber 


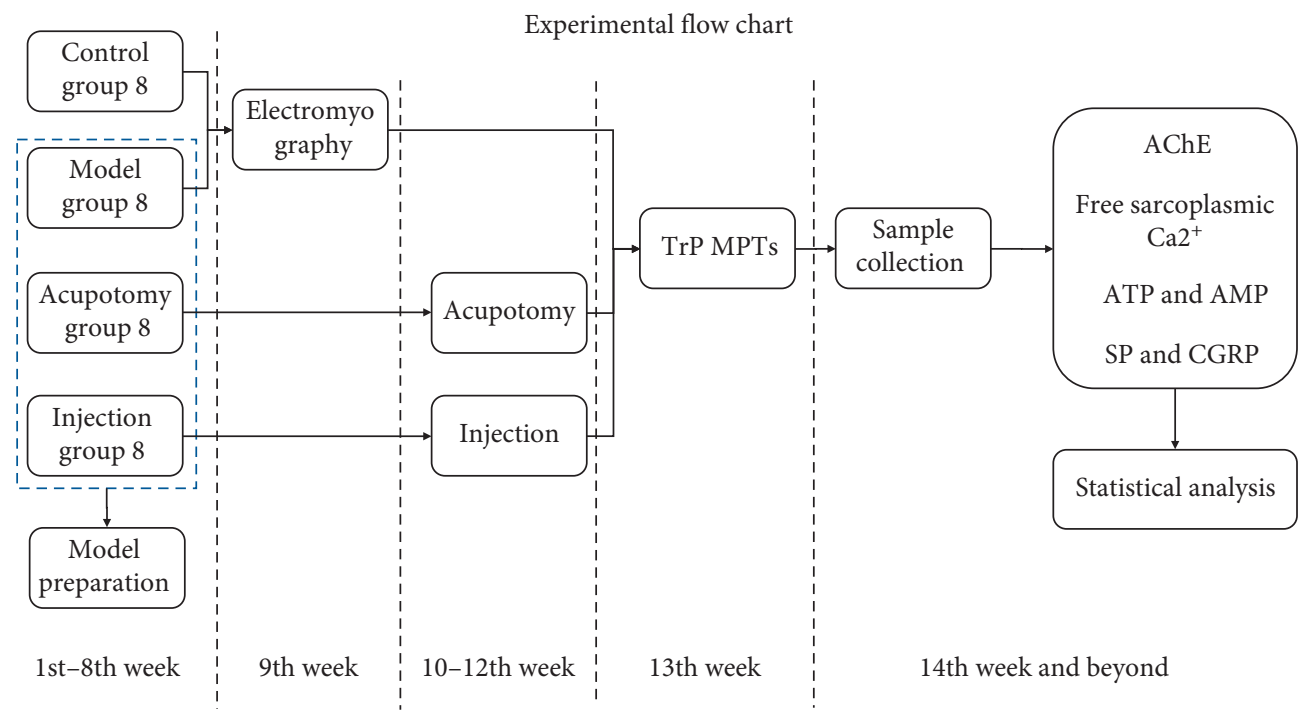

FIGURE 1: The flow chart shows procedures applied to the animals in timeline. Model preparation was in the 1st-8th week. Electromyography detection was in the 9th week. Acupotomy and injection were conducted in the 10-12th week. MPT was detected in the 13th week. Sample collection and other tests were in the 14th week and beyond.

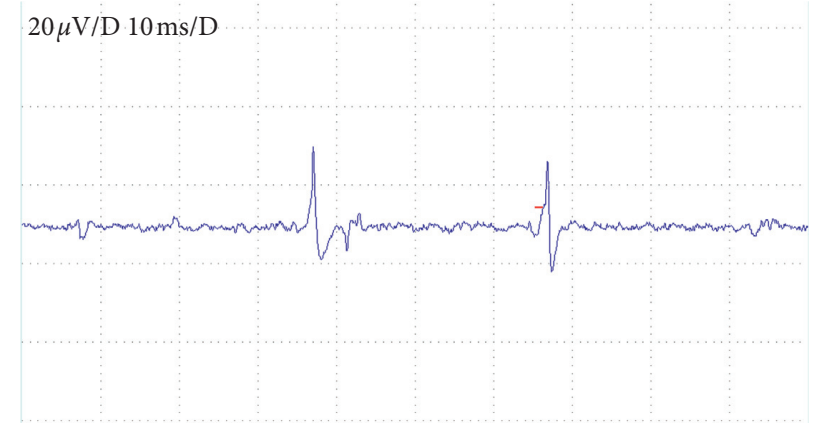

(a)

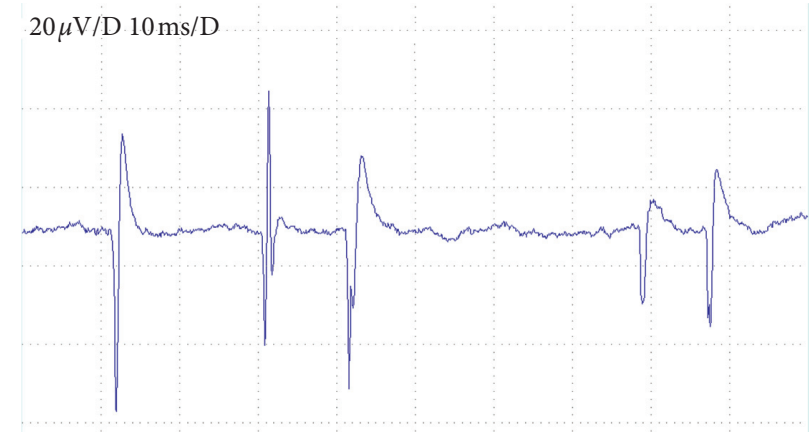

(b)

Figure 2: (a) The typical endplate potential, which turns negative from baseline. (b) The fibrillation potential, which is positive from baseline and then turns negative. The scanning speed and sensitivity are $10 \mathrm{~ms} / \mathrm{D}$ and $20 \mu \mathrm{V} / \mathrm{D}$, respectively.

contraction that consumes excessive energy. Inadequate blood flow due to continuous contraction of muscle fibers cannot effectively replenish energy and oxygen. In energy crises, $\mathrm{Ca}^{2+}$ pumps in the sarcoplasmic reticulum fail to reduce the free $\mathrm{Ca}^{2+}$ in myoplasm, aggravating muscle fiber contraction. Energy crises increase algogenic substances at local levels, which further perpetuates Ach release at motor endplates, creating a vicious circle [14].

4.2. AChE Indicates Endplate Function. This neuromuscular junction is responsible for passing nerve impulses to the skeletal muscles, causing muscle contractions. When an action potential reaches the presynaptic terminal of a motor neuron, $\mathrm{ACh}$ is released into the synaptic space where it binds to nicotinic ACh receptors (AChRs) on the muscle membrane. Binding of ACh to an AChR depolarizes the muscle fibers. $\mathrm{ACh}$ can be hydrolyzed by AChE, which is the main factor controlling the duration of ACh effects. Therefore, AChE measurement is often used to indirectly assess ACh levels.
Excessive ACh at the endplate, induced by diisopropylfluorophosphate, leads to the formation of the contracture TrP nodules [15], while injection of botulinum toxin into TrPs in New Zealand rabbits can prevent ACh release into the synaptic space, thus reducing endplate noise levels in myofascial trigger spot regions [16]. In addition, $\operatorname{TrP}$ pathogenesis is related to the sympathetic nervous system [17] through regulation of motor nerve synaptic vesicle release and levels of postsynaptic membrane AChR via its effects on the $\mathrm{Gai}_{2}-\mathrm{Hdac}_{4}-\mathrm{Myogenin}-\mathrm{MuRF}_{1}$ pathway [18].

In this study, AChE levels were decreased in the $\operatorname{TrP}$ model compared with control and acupotomy or lidocaine increased AChE levels. Similarly, a previous investigation reported that $\mathrm{ACh}$ and $\mathrm{AChR}$ levels were decreased after dry needling at precise TrPs, but AChE was increased [19]. Overall, the evidence suggests that acupotomy or dry needle treatment may affect TrPs through regulation of AChE levels in neuromuscular junctions.

In addition to inactivating $\mathrm{ACh}, \mathrm{AChE}$ also promotes neuromuscular junction survival [20]. In addition to 


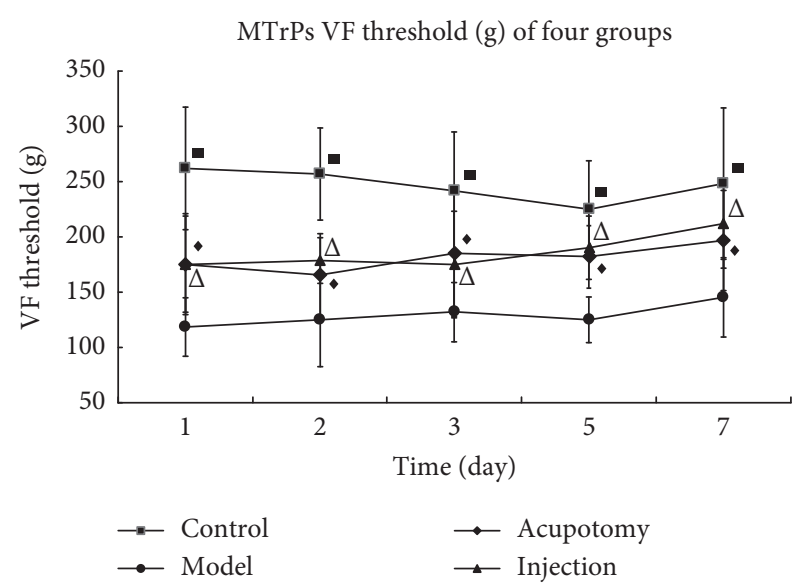

Figure 3: MPT changes (g) in each group (control, model, acupotomy, and injection; $n=8$ per group) determined by the Von Frey mechanical pain stimulator test. $P<0.05$ versus the model group. ${ }^{\Delta} P<0.05$ versus the model group. ${ }^{\star} P<0.05$ versus the model group. The error bars represent standard deviations.

reflecting the functional state of motor neurons [21], AChE activity can also indicate endplate degeneration and regeneration $[22,23]$. AChE activity is markedly decreased in denervated skeletal muscle, and the presence of AChE can be considered a marker of nerve fiber regeneration. Hence, the decrease in AChE in TrPs may indicate that TrPs are associated with changes in nerve or endplate function. Indeed, neuroaxonal degeneration and neuromuscular transmission are disordered in $\mathrm{TrP}$-containing muscles [24]. In addition, the type of muscle excitation can also regulate AChE activity in the motor endplate, suggesting that the type of synaptic transmission can also regulate AChE [25]. How continuous contraction of muscle fibers at $\operatorname{TrPs}$ is related to decreased AChE levels warrants further study.

4.3. Two Possible Reasons for Regulating Free Sarcoplasmic $\mathrm{Ca}^{2+} \cdot \mathrm{Ca}^{2+}$ is a chemical signal that can communicate messages within cells [26]. Originating from motor endplates at myofascial TrPs, spontaneous electrical activity (SEA) can reflect muscle fiber excitability, depending on $\operatorname{TrP}$ sensitivity [27]. Decreased AChE activity increases the amplitude of miniature excitatory postsynaptic currents, prolonging their attenuation time and causing sustained AChR activation that induces a $\mathrm{Ca}^{2+}$ influx [28]. Increases and decreases in free sarcoplasmic $\mathrm{Ca}^{2+}$ directly influence skeletal muscle fiber contraction and relaxation, and one important characteristic of TrPs is their continuous contraction that causes taut band formation. The $\mathrm{Ca}^{2+}$ channel blocker verapamil can effectively inhibit SEA in myofascial trigger spots in rabbit biceps femoris muscles [29]. Verapamil prevents $\mathrm{Ca}^{2+}$ influx through voltage-dependent $\mathrm{Ca}^{2+}$ channels on the plasma membrane, and it removes the inhibition of sodium $\left(\mathrm{Na}^{+}\right)$pumps caused by increased $\mathrm{Ca}^{2+}$ loads, thereby reducing intracellular $\mathrm{Ca}^{2+}$ and ensuring normal physiological cell function. This fully shows that intracellular $\mathrm{Ca}^{2+}$ content can have a significant impact on the trigger point.

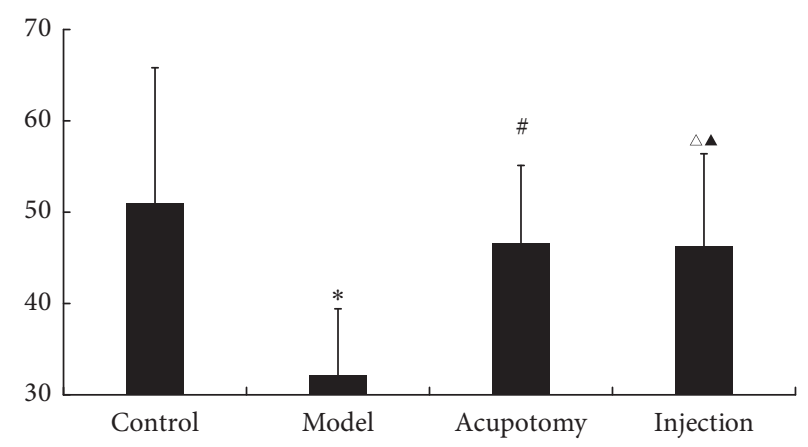

FIGURE 4: AChE concentrations (pg/mg) in each group (control, model, acupotomy, and injection; $n=8$ per group). ${ }^{*} P<0.05$ compared with the control group, ${ }^{\#} P<0.05$ compared with the model group, ${ }^{\Delta} P<0.05$ compared with the model group, and $\Delta P>0.05$ compared with the acupotomy group. The error bars represent the standard deviations.

TABle 1: Changes in AChE levels after acupotomy $(\bar{x} \pm s)$.

\begin{tabular}{lcc}
\hline Groups & $N$ & AChE $(\mathrm{pg} / \mathrm{mg})$ \\
\hline Control & 8 & $50.91 \pm 14.88$ \\
Model & 8 & $32.18 \pm 7.27^{*}$ \\
Acupotomy & 8 & $46.70 \pm 7.88^{\#}$ \\
Injection & 8 & $46.35 \pm 9.99^{\mathbf{\Delta}}$ \\
\hline
\end{tabular}

Versus control group: $P^{*}<0.05$; versus model group: $P^{\#}<0.05$; versus model group: $P^{\Delta}<0.05$; versus acupotomy group: $P^{\mathbf{\Delta}}>0.05$.

This investigation provides direct proof that free sarcoplasmic $\mathrm{Ca}^{2+}$ concentrations in the model group were significantly higher than those in the control group. Furthermore, acupotomy effectively reduced free sarcoplasmic $\mathrm{Ca}^{2+}$ in TrP muscle cells. The reason why acupotomy reduces free sarcoplasmic $\mathrm{Ca}^{2+}$ requires further study, but it may be associated with changes in $\mathrm{AChE}$ and $\mathrm{ACh}$ and/or it may be related to transient muscle cell $\mathrm{Ca}^{2+}$ levels.

Firstly, ACh release is related with muscle spindle. Stecco et al. [30] believe that if the fascia outside the muscle fiber is denser, it will hinder the shortening of the muscle spindle and even produce a chronic stretch on the muscle spindle, thus causing the continuous excitement of the muscle spindle. This explains the increased ACh from endplates at the trigger point and the continued muscle fibers contraction. Acupotomy or dry needling may reduce the excitability of muscle spindles by reducing fascia tension and ultimately reducing the ACh release and intracellular $\mathrm{Ca}^{2+}$.

Secondly, $\mathrm{Na}^{+}-\mathrm{Ca}^{2+}$ exchanger deserves attention. The $\mathrm{Na}^{+}-\mathrm{Ca}^{2+}$ exchanger NCX is an ATP-independent bidirectional transporter with two working modes. Hypoxia leads to decreased $\mathrm{Na}^{+}$/potassium $\left(\mathrm{K}^{+}\right)$-ATPase activity and increased intracellular $\mathrm{Na}^{+}$concentrations, causing NCXmediated exchange of $\mathrm{Ca}^{2+}$ into cells and consequent $\mathrm{Ca}^{2+}$ overload and damage. In a study of the effects of acupuncture on $\mathrm{Ca}^{2+}$ in skeletal muscle cells, Zhuqing et al. inferred that local acupuncture for delayed onset muscle soreness may affect NCX function, thereby inhibiting cytoplasmic $\mathrm{Ca}^{2+}$ overload [31]. 


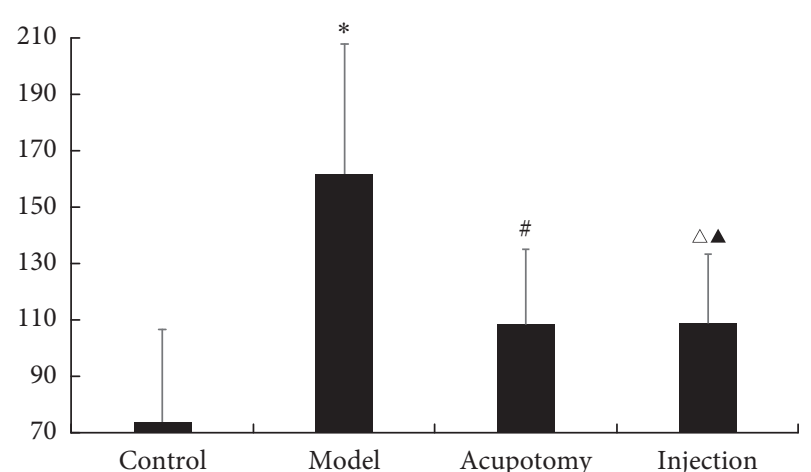

FIGURE 5: Free sarcoplasmic $\mathrm{Ca}^{2+}$ concentrations (nmol/L) in each group (control, model, acupotomy, and injection; $n=8$ per group). ${ }^{*} P<0.01$ compared with the control group, ${ }^{\#} P<0.05$ compared with the model group, ${ }^{\Delta} P<0.05$ compared with the model group, and $\Delta>0.05$ compared with the acupotomy group. The error bars represent the standard deviations.

TABLE 2: Changes in free sarcoplasmic $\mathrm{Ca}^{2+}$ levels after acupotomy $(\bar{x} \pm s)$.

\begin{tabular}{lcc}
\hline Groups & $N$ & Free sarcoplasmic $\mathrm{Ca}^{2+}(\mathrm{nmol} / \mathrm{L})$ \\
\hline Control & 8 & $74.02 \pm 32.71$ \\
Model & 8 & $161.81 \pm 46.14^{*}$ \\
Acupotomy & 8 & $105.52 \pm 26.52^{\#}$ \\
Injection & 8 & $109.1 \pm 24.41^{\mathbf{\Delta}}$ \\
\hline
\end{tabular}

Versus control group: $P^{*}<0.05$; versus model group: $P^{\#}<0.05$; versus model group: $P^{\Delta}<0.05$; versus acupotomy group: $P^{\mathbf{\Delta}}>0.05$.

4.4. ATP and ADP Indicate Energy Supply. The persistent contraction of muscle fiber TrPs increases local energy consumption and restricts blood circulation. ATP produced by oxidative phosphorylation is the direct source of energy for muscle contraction and sarcoplasmic reticulum $\mathrm{Ca}^{2+}$ reabsorption. A lack of ATP in the muscle reduces its ability to function, including both contraction and relaxation. Adenosine $5^{\prime}$-diphosphate (ADP) and AMP are degradation products of ATP and have important functions in fatigue $[32,33]$. In this study, ATP levels were lower in the model group than control group, which is consistent with the integrated hypothesis. Furthermore, this decrease was attenuated following acupotomy. This effect may be caused by improved blood circulation in TrPs in response to decreased free sarcoplasmic $\mathrm{Ca}^{2+}$.

4.5. CGRP Enhances ACh Effect at Motor Endplate. Continuous contraction leads to local ischemia and hypoxia in TrPs, which can cause the tissue to release vasoactive substances that act on nociceptors, leading to nerve sensitization, pain, and Ach release from nerve endings [34]. Using microanalytical techniques, Shah et al. found that concentrations of protons, bradykinin, CGRP, SP, tumor necrosis factor-alpha, interleukin-1 beta, serotonin, and norepinephrine were significantly higher in active $\operatorname{TrPs}[6,35,36]$. Muscle pain is associated with muscle nociceptor activation by a variety of

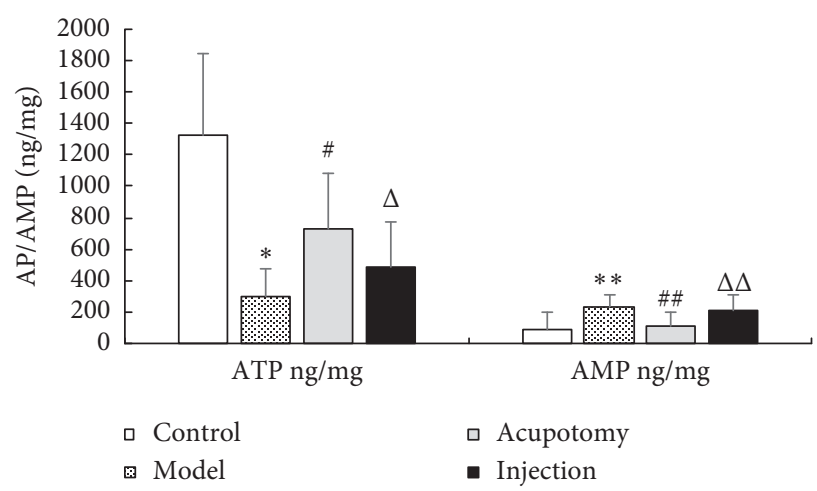

Figure 6: ATP and AMP levels (ng/mg) in each group (control, model, acupotomy, and injection; $n=8$ per group). ${ }^{*} P<0.05$ for ATP levels compared with the control group, ${ }^{\#} P<0.05$ for ATP levels compared with the model group, ${ }^{\Delta} P>0.05$ for ATP levels compared with the model group, ${ }^{* *} P<0.05$ for AMP levels compared with the control group, ${ }^{\#} P<0.05$ for AMP levels compared with the model group, and ${ }^{\Delta \Delta} P>0.05$ for AMP levels compared with the model group. The error bars represent the standard deviations.

TABle 3: Changes in ATP/AMP levels after acupotomy $(\bar{x} \pm s)$.

\begin{tabular}{lccc}
\hline Groups & $N$ & ATP $(\mathrm{ng} / \mathrm{mg})$ & AMP $(\mathrm{ng} / \mathrm{mg})$ \\
\hline Control & 8 & $1322.15 \pm 516.51$ & $85.60 \pm 114.29$ \\
Model & 8 & $298.34 \pm 181.82^{*}$ & $239.18 \pm 71.05^{* *}$ \\
Acupotomy & 8 & $734.62 \pm 344.53^{\#}$ & $108.76 \pm 89.89^{\# \#}$ \\
Injection & 8 & $490.73 \pm 280.36^{\Delta}$ & $208.49 \pm 101.65^{\Delta \Delta}$ \\
\hline
\end{tabular}

Versus control group: $P^{*}<0.05$; versus model group: $P^{\#}<0.05$; versus model group: $P^{\Delta}>0.05$; versus control group: $P^{* *}<0.05$; versus model group: $P^{\# \#}<0.05$; versus model group: $P^{\Delta \Delta}>0.05$.

endogenous substances including neuropeptides and inflammatory mediators. SP and CGRP can cause plasma extravasation, inflammation, and nociceptive effects [6]. CGRP exists in the ends of a motor neurons and promotes $\mathrm{ACh}$ release into the synaptic cleft [15]. Importantly, in relation to TrPs, CGRP increases the number of AChRs and inhibits AChE activity at motor endplate $[37,38]$, thereby enhancing the effect of ACh at the motor endplate. Gerwin and Shah hypothesized that CGRP enhances the motor endplate response to ACh by increasing the activity and synthesis of AChRs [39]. In this investigation, we found significantly elevated levels of SP and CGRP in tissue from TrPs. Furthermore, both SP and CGRP were significantly lower in the acupotomy group than the model group, which is consistent with the common observation of decreased pain after TrP treatment. Acupuncture increases local nitric oxide and microcirculation [40, 41], so local microcirculation in TrPs should be evaluated before and after needling to determine whether it dilutes algogenic substances.

In Figures 7 and 8, images of acupotomy and injection groups show lymphocyte infiltration and destruction of the fiber order, which may indicate the healing process of injuries. Because both acupotomy and injection are invasive, is the healing process of injuries related to treatment effect? 


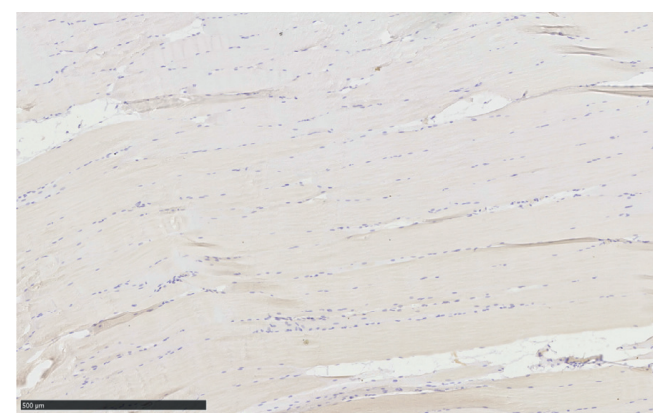

(a)

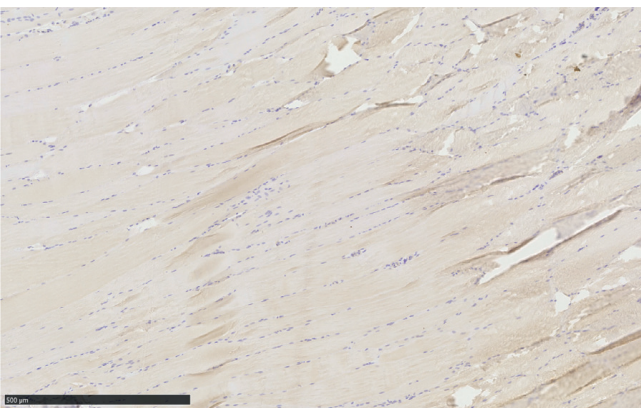

(c)

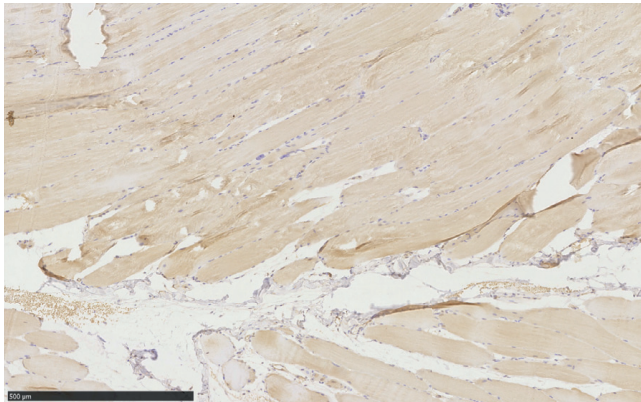

(b)

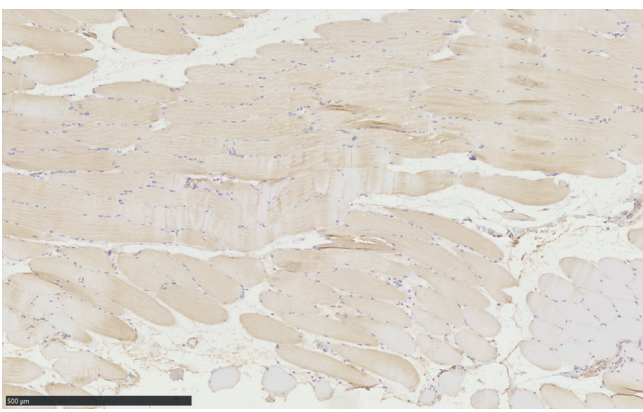

(d)

SP IOD

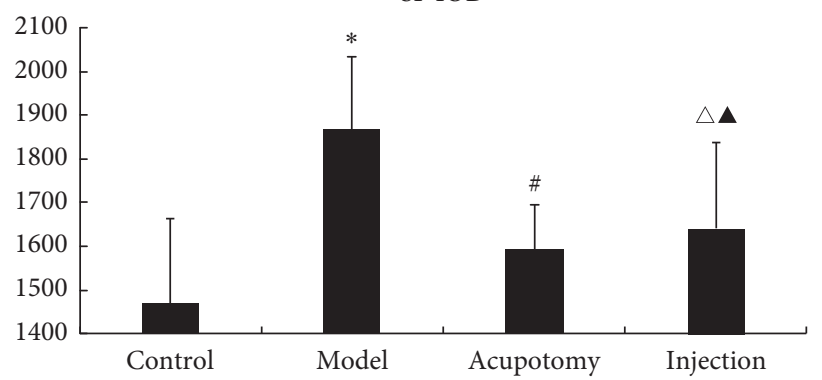

(e)

FIGURE 7: Immunohistochemistry for SP (light microscopy, $\times 200$ ). SP expression was yellowish brown in muscle fibers. SP and CGRP levels by group (control, model, acupotomy, and injection; $n=8$ per group). ${ }^{*} P<0.05$ for SP compared with the control group; ${ }^{\#} P<0.05$ for $\mathrm{SP}$ compared with the model group; ${ }^{\Delta} P<0.05$ for SP compared with the model group; ${ }^{\mathbf{A}} P>0.05$ for SP compared with the acupotomy group. The error bars represent the standard deviations. (a) Control group. (b) Model group. (c) Acupotomy group. (d) Injection group. (e) SP IOD.

TABLE 4: Changes in SP/CGRP IOD after acupotomy $(\bar{x} \pm s)$.

\begin{tabular}{lccc}
\hline Groups & $N$ & SP & CGRP \\
\hline Control & 8 & $1468 \pm 195.24$ & $1412.63 \pm 178.38$ \\
Model & 8 & $1866.38 \pm 165.38^{*}$ & $1770.3 \pm 186.90^{* *}$ \\
Acupotomy & 8 & $1591.25 \pm 102.97^{\#}$ & $1502.38 \pm 156.35^{\# \#}$ \\
Injection & 8 & $1640.75 \pm 197.48^{\Delta}$ & $1578.13 \pm 148.58^{\Delta \Delta}$ \\
\hline
\end{tabular}

Versus control group: $P^{*}<0.05$; versus model group: $P^{\#}<0.05$; versus model group: $P^{\Delta}<0.05$; versus acupotomy group: $P^{\mathbf{\Delta}}>0.05$; versus control group: $P^{* *}<0.05$; versus model group: $P^{\# \#}<0.05$; versus model group: $P^{\Delta \Delta}<0.05$; versus acupotomy group: $P^{\mathbf{\Lambda}}>0.05$.

Stecco et al. [30] believe that the increased fascia density outside the muscle fiber may excite muscle spindle, causing increased ACh from endplates and continued contraction. Acupotomy or dry/wet needling may reduce the excitability of muscle spindles by changing the inner environment around the fibers.
4.6. Relationship between $\operatorname{Tr} P$ and Ashi Points. Herman et al. proposed the concept of TrPs in 1942 [42]. Since accepting the concept of TrPs, some Chinese scholars believe that TrPs and TCM ashi points exhibit considerable overlap in their positions, needling sensation, and indications [43]. When investigations into TrPs began, TCM practitioners believed that $\operatorname{Tr} P$ s can be considered typical representative ashi points. But a contrary opinion is that the overlap of a number of pain points does not change that basic difference between acupoints and TrPs. Acupoints and TrPs are derived from vastly different concepts [44]. No matter the two concepts have similarities or differences in essence, it will be an hint to promote the reseach of ashi points in TCM. Although ashi points have been understood by practitioners of TCM for several centuries and various acupuncture methods have been applied in TCM to treat ashi points, deep research into ashi points has been lacking. 


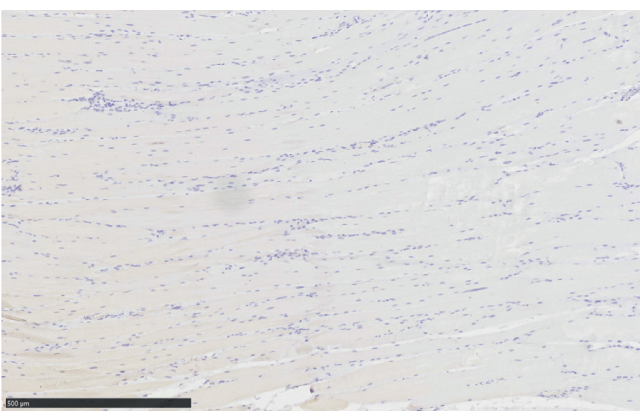

(a)

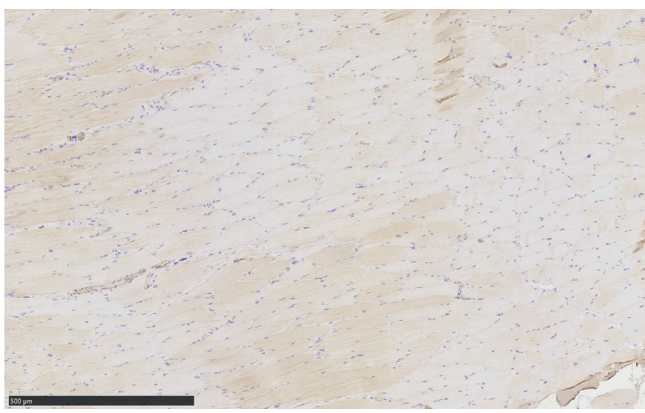

(c)

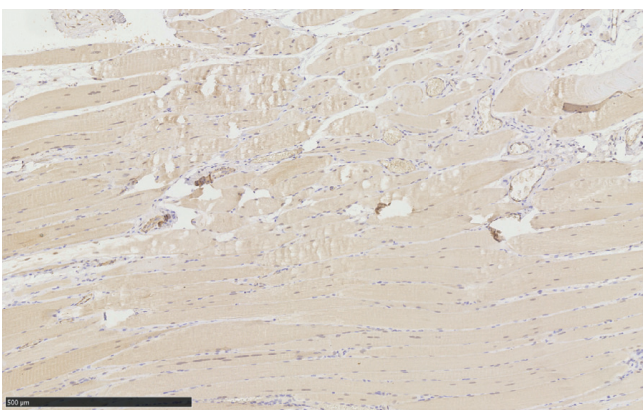

(b)

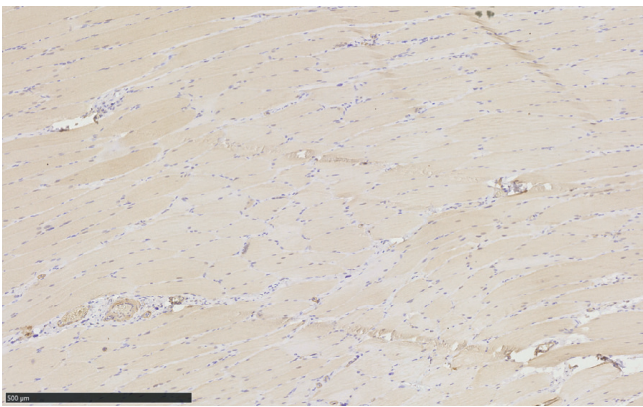

(d)

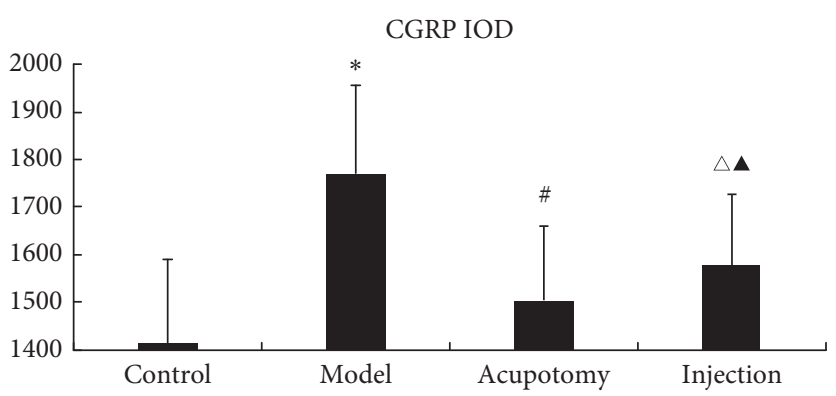

(e)

FIGURE 8: Immunohistochemistry for CGRP (light microscopy, $\times 200$ ). CGRP expression was yellowish brown in muscle fibers. CGRP levels were significantly higher in the model group than the control $(P<0.05)$, acupotomy $(P<0.05)$, and injection $(P<0.05)$ groups. There was no significant difference in CGRP content between the acupotomy and injection groups $(P>0.05)$. SP and CGRP levels by group (control, model, acupotomy, and injection; $n=8$ per group). ${ }^{*} P<0.05$ for CGRP compared with the control group; ${ }^{\#} P<0.05$ for CGRP compared with the model group; ${ }^{\wedge} P>0.05$ for CGRP compared with the acupotomy group; ${ }^{\wedge} P<0.05$ for CGRP compared with the model group. The error bars represent the standard deviations. (a) Control group. (b) Model group. (c) Acupotomy group. (d) Injection group. (e) CGRP IOD.

\section{Conclusion}

Developed from ancient acupuncture techniques, acupotomy is similar to dry needling; both require accurate insertion of needles into local TrPs, and they may also work via similar mechanisms. The observations from our study support the hypothesis that energy crises affect TrP muscle fibers. Furthermore, our data demonstrate that acupotomy can influence TrP MPTs and levels of AChE, free sarcoplasmic $\mathrm{Ca}^{2+}$, ATP, AMP, SP, and CGRP. Its effects were similar to local lidocaine injection, except only acupotomy affected ATP and AMP levels. It is indicated that acupotomy has effect on trigger point pathophysiology, which is different from acupuncture analgesia. Future research will focus on the mechanism by which acupotomy resolves energy crises in TrPs, peripheric or central?
The general understanding of acupuncture mechanism is that the mechanical signals generated by needle insertion are converted into nerve signals through the acupoint and transmitted to the central nervous system and on to the efferent system and effector organs via the neuro-endocrineimmune system [45]. Further, acupuncture may have peripheral effects, independent of overall neurohumoral regulation [46, 47]. Zhuqing et al. [48] found that local acupuncture is effective in delaying muscle soreness onset because it adjusts the catabolism and anabolism of muscle contractile proteins. This differs from the mechanism involved in acupuncture analgesia. The same effect was observed in isolated semitendinosus muscle experiments, suggesting that acupuncture may have a peripheral mechanism independent of overall neurohumoral regulation. For visceral disorders, it is necessary for acupuncture to work via 
the neuro-endocrine-immune system because the viscera cannot be needled directly. But for myofascial lesions, it is different because muscular fasciae can be needled directly. For myofascial disorder, what will happen when it is needled directly? Acupuncture was shown to trigger a local increase in adenosine in human subjects $[49,50]$ and to increase local microcirculation $[40,41]$. It is still controversial whether a trigger point is a central or peripheral phenomenon [11]. Accurate positioning at TrPs is an important requirement for both acupotomy and dry needling, suggesting that the effects of these procedures may have similar underlying peripheral mechanisms; however, a review reported that due to the scarcity of reliable studies, the current evidence on the local effects of acupuncture is insufficient to draw reliable conclusions [51]. Additional investigations are needed to verify the local effects of acupuncture.

The limitation of this study is that only one method was used to test each variable; therefore, our data lack verification with multiple experimental methods. And several anesthesia procedures during the experiment may influence the results.

\section{Data Availability}

The data used to support the findings of this study are available from the corresponding author upon request.

\section{Conflicts of Interest}

The authors declare that there are no conflicts of interest regarding the publication of this paper.

\section{Acknowledgments}

The research program was funded by the National Natural Science Foundation of China (no. 81503653).

\section{References}

[1] S. Money, "Pathophysiology of trigger points in myofascial pain syndrome," Journal of Pain \& Palliative Care Pharmacotherapy, vol. 31, no. 2, pp. 158-159, 2017.

[2] C. K. Chen and A. J. Nizar, "Myofascial pain syndrome in chronic back pain patients," The Korean Journal of Pain, vol. 24, no. 2, pp. 100-104, 2011.

[3] D. G. Simons and J. Travell, "Myofascial trigger points, a possible explanation,” Pain, vol. 10, pp. 106-109, 1981.

[4] D. G. Simons, "New views of myofascial trigger points: etiology and diagnosis," Archives of Physical Medicine and Rehabilitation, vol. 89, no. 1, pp. 157-159, 2008.

[5] Q.-M. Huang, G. Ye, Z.-Y. Zhao, J.-J. Lv, and L. Tang, "Myoelectrical activity and muscle morphology in a rat model of myofascial trigger points induced by blunt trauma to the vastus medialis," Acupuncture in Medicine, vol. 31, no. 1, pp. 65-73, 2013.

[6] J. P. Shah and E. A. Gilliams, "Uncovering the biochemical milieu of myofascial trigger points using in vivo microdialysis: an application of muscle pain concepts to myofascial pain syndrome," Journal of Bodywork and Movement Therapies, vol. 12, no. 4, pp. 371-384, 2008.

[7] S. Li, T. Shen, Y. Liang, B. Bai, and Y. Zhang, "Miniscalpelneedle treatment is effective for work-related neck and shoulder musculoskeletal disorders," Evidence-Based Complementary and Alternative Medicine, vol. 2016, Article ID 5760240, 9 pages, 2016.

[8] J. Y. Ro, N. Capra, and R. Masri, "Development of a behavioral assessment of craniofacial muscle pain in lightly anesthetized rats," Pain, vol. 104, no. 1-2, pp. 179-185, 2003.

[9] J. Y. Ro and N. F. Capra, "Assessing mechanical sensitivity of masseter muscle in lightly anesthetized rats: a model for craniofacial muscle hyperalgesia," Neuroscience Research, vol. 56, no. 1, pp. 119-123, 2006.

[10] G. Grynkiewicz, M. Poenie, and R. Y. Tsien, “A new generation of $\mathrm{Ca}^{2+}$ indicators with greatly improved fluorescence properties," The Journal of Biological Chemistry, vol. 260, no. 6, pp. 3440-3450, 1985.

[11] C. Fernández-de-las-Peñas and J. Dommerholt, "Myofascial trigger points: peripheral or central phenomenon?," Current Rheumatology Reports, vol. 16, no. 1, p. 395, 2014.

[12] M. J. L. Hocking, "Exploring the central modulation hypothesis: do ancient memory mechanisms underlie the pathophysiology of trigger points?," Current Pain and Headache Reports, vol. 17, no. 7, 2013.

[13] H.-Y. Ge, C. Fernández-de-las-Peñas, P. Madeleine, and L. Arendt-Nielsen, "Topographical mapping and mechanical pain sensitivity of myofascial trigger points in the infraspinatus muscle," European Journal of Pain, vol. 12, no. 7, pp. 859-865, 2008.

[14] E. Vazquez-Delgado, J. Cascos-Romero, and C. Gay-Escoda, "Myofascial pain syndrome associated with trigger points: a literature review. (I): epidemiology, clinical treatment and etiopathogeny," Medicina Oral Patología Oral y Cirugia Bucal, vol. 14, no. 10, pp. e494-e498, 2009.

[15] S. Mense, D. G. Simons, U. Hoheisel, and B. Quenzer, "Lesions of rat skeletal muscle after local block of acetylcholinesterase and neuromuscular stimulation," Journal of Applied Physiology, vol. 94, no. 6, pp. 2494-2501, 2003.

[16] T.-S. Kuan, J.-T. Chen, S.-M. Chen, C.-H. Chien, and C.-Z. Hong, "Effect of botulinum toxin on endplate noise in myofascial trigger spots of rabbit skeletal muscle," American Journal of Physical Medicine \& Rehabilitation, vol. 81, no. 7, pp. 512-520, 2002.

[17] J.-T. Chen, S.-M. Chen, T.-S. Kuan, K.-C. Chung, and C.-Z. Hong, "Phentolamine effect on the spontaneous electrical activity of active loci in a myofascial trigger spot of rabbit skeletal muscle," Archives of Physical Medicine and Rehabilitation, vol. 79, no. 7, pp. 790-794, 1998.

[18] A. C. Z. Rodrigues, M. L. Messi, Z. M. Wang et al., "The sympathetic nervous system regulates skeletal muscle motor innervation and acetylcholine receptor stability," Acta Physiologica, vol. 225, no. 3, Article ID e13195, 2019.

[19] Q. G. Liu, L. Liu, Q. M. Huang, T. T. Nguyen, Y. T. Ma, and J. M. Zhao, "Decreased spontaneous electrical activity and acetylcholine at myofascial trigger spots after dry needling treatment: a pilot study," Evidence-Based Complementary and Alternative Medicine, vol. 2017, Article ID 3938191, 7 pages, 2017.

[20] M. L. Campanari, M. S. Garcia-Ayllon, S. Ciura, J. Saez-Valero, and E. Kabashi, "Neuromuscular junction impairment in amyotrophic lateral sclerosis: reassessing the role of acetylcholinesterase," Frontiers in Molecular Neuroscience, vol. 9, 2016.

[21] E. Arikawa-Hirasawa, S. G. Rossi, R. L. Rotundo, and Y. Yamada, "Absence of acetylcholinesterase at the neuromuscular junctions of perlecan-null mice," Nature Neuroscience, vol. 5, no. 2, pp. 119-123, 2002.

[22] I. Martinez-Pena y Valenzuela, R. I. Hume, E. Krejci, and M. Akaaboune, "In vivo regulation of acetylcholinesterase 
insertion at the neuromuscular junction," Journal of Biological Chemistry, vol. 280, no. 36, pp. 31801-31808, 2005.

[23] I. Martinez-Pena y Valenzuela and M. Akaaboune, "Acetylcholinesterase mobility and stability at the neuromuscular junction of living mice," Molecular Biology of the Cell, vol. 18, no. 8, pp. 2904-2911, 2007.

[24] C.-W. Chang, Y.-R. Chen, and K.-F. Chang, "Evidence of neuroaxonal degeneration in myofascial pain syndrome: a study of neuromuscular jitter by axonal microstimulation," European Journal of Pain, vol. 12, no. 8, pp. 1026-1030, 2008.

[25] R. Gašperšič, B. Koritnik, N. Črne-Finderle, and J. Sketelj, "Acetylcholinesterase in the neuromuscular junction," Chemico-Biological Interactions, vol. 119-120, pp. 301-308, 1999.

[26] J. C. Calderón, P. Bolaños, and C. Caputo, "The excitationcontraction coupling mechanism in skeletal muscle," Biophysical Reviews, vol. 6, no. 1, pp. 133-160, 2014.

[27] H.-Y. Ge, C. Fernández-de-las-Peñas, and S.-W. Yue, "Myofascial trigger points: spontaneous electricalactivity and its consequences for pain inductionand propagation," Chinese Medicine, vol. 6, no. 1, p. 13, 2011.

[28] C. C. Garcia, J. G. Potian, K. Hognason et al., "Acetylcholinesterase deficiency contributes to neuromuscular junction dysfunction in type 1 diabetic neuropathy," American Journal of Physiology-Endocrinology and Metabolism, vol. 303, no. 4, pp. E551-E561, 2012.

[29] C.-R. Hou, K.-C. Chung, J.-T. Chen, and C.-Z. Hong, "Effects of a calcium channel blocker on electrical activity in myofascial trigger spots of rabbits," American Journal of Physical Medicine \& Rehabilitation, vol. 81, no. 5, pp. 342-349, 2002.

[30] A. Stecco, M. Gesi, C. Stecco, and R. Stern, "Fascial components of the myofascial pain syndrome," Current Pain and Headache Reports, vol. 17, no. 8, p. 352, 2013.

[31] Q. Zhuqing, L. Dinghou, and W. Yirun, "Effects of acupuncture on intracellular calcium distribution during skeletal muscle injury and its mechanism," Chinese Journal of Sports Medicine, vol. 1, pp. 7-12, 1995.

[32] X. Ling, "Ca ${ }^{2+}, \mathrm{ADP}, \mathrm{Pi}$ and sport fatigue," Journal of Beijing Sport University, vol. 29, no. 3, p. 363, 2006.

[33] L. N. Jia Danbing, Fatigue, Academe Press, Beijing, China, 2009.

[34] E. A. Bukharaeva, R. I. Salakhutdinov, F. Vyskočil, and E. E. Nikolsky, "Spontaneous quantal and non-quantal release of acetylcholine at mouse endplate during onset of hypoxia," Physiological Research, vol. 54, no. 2, pp. 251-255, 2005.

[35] J. P. Shah, T. M. Phillips, J. V. Danoff, and L. H. Gerber, "An in vivo microanalytical technique for measuring the local biochemical milieu of human skeletal muscle," Journal of Applied Physiology, vol. 99, no. 5, pp. 1977-1984, 2005.

[36] J. P. Shah, J. V. Danoff, M. J. Desai et al., "Biochemicals associated with pain and inflammation are elevated in sites near to and remote from active myofascial trigger points," Archives of Physical Medicine and Rehabilitation, vol. 89, no. 1, pp. 16-23, 2008.

[37] H. L. Fernandez, M. Chen, I. Nadelhaft, and J. A. Durr, "Calcitonin gene-related peptides: their binding sites and receptor accessory proteins in adult mammalian skeletal muscles," Neuroscience, vol. 119, no. 2, pp. 335-345, 2003.

[38] C. A. Hodges-Savola and H. L. Fernandez, "A role for calcitonin gene-related peptide in the regulation of rat skeletal muscle G4 acetylcholinesterase," Neuroscience Letters, vol. 190, no. 2, pp. 117-120, 1995.

[39] R. D. Gerwin, J. Dommerholt, and J. P. Shah, "An expansion of Simons' integrated hypothesis of trigger point formation,"
Current Pain and Headache Reports, vol. 8, no. 6, pp. 468-475, 2004.

[40] M. Tsuchiya, E. F. Sato, M. Inoue, and A. Asada, "Acupuncture enhances generation of nitric oxide and increases local circulation," Anesthesia \& Analgesia, vol. 104, no. 2, pp. 301-307, 2007.

[41] S. Min, H. Lee, S.-Y. Kim et al., "Local changes in microcirculation and the analgesic effects of acupuncture: a laser Doppler perfusion imaging study," The Journal of Alternative and Complementary Medicine, vol. 21, no. 1, pp. 46-52, 2015.

[42] J. T. S. R. M. Herman, "Pain and disability of the shoulder and arm," Journal of the American Medical Association, vol. 120, no. 6, pp. 417-422, 1942.

[43] G.-F. Yang, C.-N. Ji, and S. Q. Yuan, "Modern medical explanation on Ashi points," Chinese Acupuncture \& Moxibustion, vol. 32, no. 2, pp. 180-182, 2012.

[44] S. Birch, "On the impossibility of trigger point-acupoint equivalence: a commentary on Peter Dorsher's analysis," The Journal of Alternative and Complementary Medicine, vol. 14, no. 4, pp. 343-345, 2008.

[45] N. C. Li, M. Y. Li, B. Chen, and Y. Guo, "A new perspective of acupuncture: the interaction among three networks leads to neutralization," Evidence-Based Complementary and Alternative Medicine, vol. 2019, Article ID 2326867, 10 pages, 2019.

[46] J. Chu, "The local mechanism of acupuncture," Chinese Medical Journal (Taipei), vol. 65, no. 7, pp. 299-302, 2002.

[47] Y. Zhang and C. Guo, "Direct effects of acupuncture on local lesion," China Journal of Traditional Chinese Medicine and Pharmacy, vol. 31, no. 10, pp. 3926-3930, 2016.

[48] Q. Zhuqing, L. Dinghou, and W. Yirun, "Discussion on the effect and mechanism of acupuncture on promoting recovery of muscles with exhaustion and delayed structural changesobservation on muscle strength and ultrastructure," Journal of Beijing Institute of Physical Education, vol. 2, pp. 38-47, 1993.

[49] T. Takano, X. Chen, F. Luo et al., "Traditional acupuncture triggers a local increase in adenosine in human subjects," The Journal of Pain, vol. 13, no. 12, pp. 1215-1223, 2012.

[50] N. Goldman, M. Chen, T. Fujita et al., "Adenosine A1 receptors mediate local anti-nociceptive effects of acupuncture," Nature Neuroscience, vol. 13, no. 7, pp. 883-888, 2010.

[51] S.-Y. Kim, S. Min, H. Lee et al., "Changes of local blood flow in response to acupuncture stimulation: a systematic review," Evidence-Based Complementary and Alternative Medicine, vol. 2016, Article ID 9874207, 11 pages, 2016. 